\title{
And the story goes on: non-conventional dysplasia of the colorectum
}

\author{
Lavisha S. Punjabi, Yi Neng Lai, Anjula Thomas \\ Department of Anatomical Pathology, Sengkang General Hospital, Singapore
}

The adenoma-carcinoma sequence of carcinogenesis in the colorectum, featuring the progressive accumulation of alterations of $A P C, K R A S, p 53$, and other genes, was elucidated in the 1990s and has since become a textbook example of the stepwise model of carcinogenesis. In recent decades, our understanding of colorectal dysplasia has expanded through the study of the clinical, morphologic, molecular aspects of serrated dysplasia and non-conventional dysplasia in inflammatory bowel disease (IBD). Hence, we read with interest Choi's timely review of the seven morphologic subtypes of non-conventional dysplasia in IBD [1].

The assessment of dysplasia in the context of IBD is a widely accepted and long-held area of diagnostic difficulty, associated with fair to moderate interobserver agreement even among subspeciality pathologists [2]. The question that naturally arises from the discovery of these unusual morphologic subtypes of dysplasia, that was to some extent based on retrospective study and generous resection specimens, is its applicability and impact on routine reporting of limited endoscopic biopsies. It is plausible that awareness of these morphologic subtypes could facilitate more accurate histologic recognition of dysplasia.

Alternatively, the devil's advocate may posit that the subtlety of some of these morphologic subtypes may make the diagnosis of dysplasia in superficial biopsies inconclusive and ambiguous. Of note, hypermucinous dysplasia shows progressively less cytologic atypia towards the surface and is thus a more subtle form of dysplasia than we are accustomed to recognizing. Contrary to its low-grade appearance however, this subtype harbors a higher

Received: October 13, 2021 Revised: December 27, 2021

Accepted: December 28, 2021

Corresponding Author: Lavisha S. Punjabi, MBBS

Department of Anatomical Pathology, Sengkang General Hospital, 110 Sengkang E

Way, Singapore 544886

Tel: +65-6930-5000, E-mail: lavisha.s.punjabi@gmail.com rate of KRAS alterations and aneuploidy [3,4], rendering it a high-risk lesion that should not be missed. From the clinical point of view, it would therefore be important to recognize nonconventional dysplasia, particularly the high-risk subtypes, and recommend short-term follow-up for these lesions, despite the concern about the ambiguous diagnosis for dysplasia. Conversely, from the healthcare systems point of view, a higher overall "indefinite for dysplasia" call rate may result in increased healthcare costs, particularly if the number needed to treat is high. Evidently, prospective evaluation of the impact on and of reporting, including inter-observer and intra-observer reproducibility among both subspecialty and general pathologists, is required, albeit conceivably limited by relatively small case numbers.

As with many areas in pathology, new knowledge often undergoes a process of "splitting," investigation and then meaningful "lumping." A prime example of this process, although out of the field of gastrointestinal pathology, is the study of subtypes of non-mucinous adenocarcinoma of the lung, that began with meticulous morphologic descriptions, such as lepidic, acinar, complex glandular, papillary, micropapillary and solid. Subsequent data on the prognostic implications of each of these subtypes facilitated meaningful grouping within a three-tier grading system formulated by the International Association for the Study of Lung Cancer, incorporated in the latest iteration of the World Health Organization classification of thoracic tumors. Similarly, further study of the behavior of the morphologic subtypes of non-conventional dysplasia in the colorectum may eventually allow formulation of broader categories in the future. In addition, one may contend that serrated lesions in IBD and sporadic serrated lesions could be united, given the similarity in clinical, pathologic, and molecular features.

As a matter of topical interest and therapeutic relevance, findings of a recent study by Kim et al. [5] concluded that NTRK-rearranged colorectal carcinomas progress exclusively via the serrat- 
ed pathway of neoplasia, thus expanding the molecular landscape of serrated colorectal lesions. To the best of our knowledge, oncogenic gene fusions including NTRK fusions have yet to be studied in serrated lesions in IBD, and would indeed form an interesting research question.

In conclusion, while we have made strides in expanding the concepts of colorectal dysplasia, the present-day story of colorectal carcinogenesis in IBD is an unfinished one, with many curious questions, practical and scientific, yet to be solved.

\section{Ethics Statement}

Not applicable.

\section{Availability of Data and Material}

Data sharing not applicable to this article as no datasets were generated or analyzed during the study.

\section{Code Availability}

Not applicable.

\section{ORCID}

Lavisha S. Punjabi https://orcid.org/0000-0003-0479-1690

\section{Author Contributions}

Conceptualization: LSP. Writing-original draft: LSP. Writing-review \& editing: YNL, AT. Approval of final manuscript: all authors.

\section{Conflicts of Interest}

The authors declare that they have no potential conflicts of interest.

\section{Funding Statement}

No funding to declare.

\section{References}

1. Choi WT. Non-conventional dysplastic subtypes in inflammatory bowel disease: a review of their diagnostic characteristics and potential clinical implications. J Pathol Transl Med 2021; 55: 83-93.

2. Laine L, Kaltenbach T, Barkun A, et al. SCENIC international consensus statement on surveillance and management of dysplasia in inflammatory bowel disease. Gastrointest Endosc 2015; 81: 489501

3. Lee H, Rabinovitch PS, Mattis AN, Lauwers GY, Choi WT. Nonconventional dysplasia in inflammatory bowel disease is more frequently associated with advanced neoplasia and aneuploidy than conventional dysplasia. Histopathology 2021; 78: 814-30.

4. Andersen SN, Lovig T, Clausen OP, Bakka A, Fausa O, Rognum TO. Villous, hypermucinous mucosa in long standing ulcerative colitis shows high frequency of K-ras mutations. Gut 1999; 45: 686-92.

5. Kim JH, Hong JH, Choi YL, et al. NTRK oncogenic fusions are exclusively associated with the serrated neoplasia pathway in the colorectum and begin to occur in sessile serrated lesions. J Pathol 2021; 255: 399-411. 